Journal of Applied Mathematics and Stochastic Analysis, 16:2 (2003), 141-161. Printed in the USA (C) 2003 by North Atlantic Science Publishing Company

\title{
FUNCTIONAL INTEGRO-DIFFERENTIAL STOCHASTIC EVOLUTION EQUATIONS IN HILBERT SPACE
}

\author{
DAVID N. KECK \\ Ohio University, Department of Mathematics \\ 321 Morton Hall, Athens, $\mathrm{OH}$ 45701, USA \\ and \\ MARK A. McKIBBEN ${ }^{1}$ \\ Goucher College, Department of Mathematics and Computer Science, \\ 1021 Dulaney Valley Road, Baltimore, MD 21204, USA
}

(Received September 2002; Revised February 2003)

\begin{abstract}
We investigate a class of abstract functional integro-differential stochastic evolution equations in a real separable Hilbert space. Global existence results concerning mild and periodic solutions are formulated under various growth and compactness conditions. Also, related convergence results are established and an example arising in the mathematical modeling of heat conduction is discussed to illustrate the abstract theory.

Key words: Stochastic evolution equations, semi-group, Wiener process, probability measure.
\end{abstract}

AMS (MOS) subject classification: 34K30, 34F05, 60H10

\section{Introduction}

The purpose of this paper is to study the global existence and convergence properties of mild solutions to a class of abstract semi-linear functional stochastic integro-differential equations of the general form

$$
\begin{gathered}
x^{\prime}(t)=A x(t)+F(x)(t)+\int_{0}^{t} G(x)(s) d W(s), \quad 0 \leq t \leq T, \\
x(0)=h(x)+x_{0},
\end{gathered}
$$

in a real separable Hilbert space $H$. Here, $A: D(A) \subset H \rightarrow H$ is a linear (possibly unbounded) operator, $G: \mathcal{C}([0, T] ; H) \rightarrow C\left([0, T] ; L^{2}(\Omega ; B L(K ; H))\right)$ (where $K$ is a

\footnotetext{
${ }^{1}$ This work was begun during the author's visit to Ohio University in June 2002, a trip which was supported by a summer research grant awarded by the Goucher College Alumnae and Alumni Junior Faculty Fund.
} 
real separable Hilbert space), $F: \mathcal{C}([0, T] ; H) \rightarrow L^{p}\left(0, T ; L^{2}(\Omega ; H)\right)(1 \leq p<\infty), W$ is a $K$-valued Wiener process with incremental covariance described by the nuclear operator $Q, x_{0}$ is an $\mathcal{F}_{0}$-measurable $H$-valued random variable independent of $W$, and $h: \mathcal{C}([0, T] ; H) \rightarrow L_{0}^{2}(\Omega ; H)$.

The present work may be regarded as a direct attempt to extend recent results developed in $[7,10,16,18,20]$ to a broader class of functional stochastic equations. The equations considered in the aforementioned papers can be viewed as special cases of (1.1) by making the appropriate identifications of $F, G$, and $h$. Moreover, we further extend these results by incorporating more general initial conditions. In particular, mild periodic solutions are obtained. To the authors' knowledge the results in this paper are new even in the case of a classical initial condition (i.e., when $h=0$ ).

The deterministic version of (1.1) (and related equations) coupled with a classical initial condition has been studied extensively both when $A$ is linear and when $A$ is nonlinear. We refer the reader to $[8,30]$ and the references therein. Byszewski [13] introduced nonlocal initial conditions into such abstract initial-value problems and argued that the corresponding models more accurately describe the phenomena since more information was taken into account at the onset of the experiment, thereby reducing the ill effects incurred by a single (possibly erroneous) initial measurement. Since then, many authors have continued this work in several directions and established existence theories for first-order nonlinear evolution equations [2, 4, 29], second-order equations [7], delay equations [7, 28], Volterra integral and integro-differential equations [5, 25], and differential inclusions [1]. Concrete nonlocal parabolic and elliptic partial (integro-) differential equations arising in the mathematical modeling of various physical, biological, and ecological phenomena, as well as a discussion of the advantages of replacing the classical initial condition with a more general functional, can be found in $[13,21]$ and the references contained therein.

Stochastic differential equations (SDEs) in both finite and infinite dimensions have also received considerable attention. We refer the reader to $[10,32]$ for a thorough discussion in the finite dimensional setting, and [14, 19] for the infinite dimensional setting. A semi-group-theoretic development of a theory for the stochastic analogues of deterministic evolution equations is both powerful and beneficial since it enables one to investigate a broad class of stochastic partial differential equations within a unified context. SDEs are important from the viewpoint of applications since they incorporate (natural) randomness into the mathematical description of the phenomena, and, therefore, provide a more accurate description of it. Moreover, coupling such equations with a nonlocal initial condition strengthens the model even further.

The basic tools used in this paper include fixed-point techniques, the theory of (compact) linear semi-groups, results for probability measures, and methods and results for infinite dimensional SDEs. The results are important from the viewpoint of applications since they cover nonlocal generalizations of integro-differential SDEs arising in fields such as electromagnetic theory, population dynamics, and heat conduction in materials with memory $[10,17,19,32]$.

The outline of the paper is as follows. We review some basic facts about linear semi-groups, the theory of SDEs, and probability measures in Section 2. Then, Sections 3 and 4 are devoted to the development of our main existence results, while a discussion of various convergence results immediately follows in Section 5. Finally, the paper concludes with a discussion of a concrete nonlocal integro-partial SDE in Section 6. 


\section{Preliminaries}

For further background of this section, we refer the reader to $[9,11,12,14,15,19,23$, $30,32]$. Throughout this manuscript, $H$ and $K$ denote real separable Hilbert spaces equipped with norms $\|\cdot\|_{H}$ and $\|\cdot\|_{K}$, respectively, and the space of bounded linear operators from $K$ to $H$ is denoted by $B L(K ; H)$ (or simply $B L(H)$ if $K=H$ ). Also, for Banach spaces $X$ and $Y$, the space of continuous functions from $X$ into $Y$ (equipped with the usual sup-norm) shall be denoted by $C(X ; Y)$, while $L^{p}(0, T ; X)$ shall represent the space of $X$-valued functions that are $p$-integrable on $[0, T]$.

Let $(\Omega, \mathcal{F}, P)$ be a complete probability space equipped with a normal filtration $\left\{\mathcal{F}_{t}: 0 \leq t \leq T\right\}$ (i.e., a right-continuous, increasing family of sub $\sigma$-algebras of $\mathcal{F}$ ). An $H$-valued random variable is an $\mathcal{F}$-measurable function $X: \Omega \rightarrow H$ and a collection of random variables $S=\{X(t ; \omega): \Omega \rightarrow H \mid 0 \leq t \leq T\}$ is called a stochastic process. Henceforth, we shall suppress the dependence on $\omega \in \Omega$ and write $X(t)$ instead of $X(t ; \omega)$ and $X:[0, T] \rightarrow H$ in place of $S$.

The collection of all strongly-measurable, square-integrable $H$-valued random variables, denoted by $L^{2}(\Omega ; H)$, is a Banach space equipped with norm $\|X(\cdot)\|_{L^{2}(\Omega ; H)}=$ $\left(E\|X(\cdot ; \omega)\|_{H}^{2}\right)^{1 / 2}$, where the expectation, $E$, is defined by $E(g)=\int_{\Omega} g(\omega) d P$. An important subspace is given by $L_{0}^{2}(\Omega ; H)=\left\{f \in L^{2}(\Omega ; H): f\right.$ is $\mathcal{F}_{0}$-measurable $\}$. Next, we define the space $\mathcal{C}([0, T] ; H)$ to be the set $\left\{v \in C\left([0, T] ; L^{2}(\Omega ; H)\right): v\right.$ is $\mathcal{F}_{t}$-adapted $\}$. One can prove that this is a Banach space when equipped with the norm

$$
\|v\|_{\mathcal{C}}=\sup _{0 \leq t \leq T}\left(E\|v(t)\|_{H}^{2}\right)^{1 / 2}
$$

Definition 2.1: A stochastic process $\{W(t): t \geq 0\}$ in a real separable Hilbert space $H$ is a Wiener process if for each $t \geq 0$,

(i) $W(t)$ has continuous sample paths and independent increments,

(ii) $W(t) \in L^{2}(\Omega ; H)$ and $E(W(t))=0$,

(iii) $\operatorname{Cov}(W(t)-W(s))=(t-s) Q$, where $Q \in B L(K ; H)$ is a nonnegative nuclear operator.

Consider the initial-value problem

$$
\begin{gathered}
x^{\prime}(t)=A x(t)+f(t)+g(t) W^{\prime}(t), \quad 0 \leq t \leq T, \\
x(0)=x_{0},
\end{gathered}
$$

where $A: H \rightarrow H$ generates a $C_{0}$-semi-group $\{S(t): t \geq 0\}$ on $H, f \in L^{1}(0, T ; H)$, $g \in B L(K ; H), W$ is a $K$-valued Wiener process with respect to $\left\{\mathcal{F}_{t}: 0 \leq t \leq T\right\}$, and $x_{0} \in L_{0}^{2}(\Omega ; H)$.

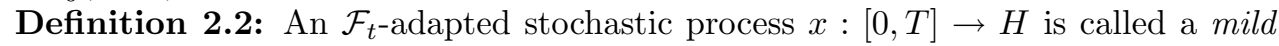
solution of $(2.2)$ if $x(t)$ is measurable, for all $t \in[0, T], \int_{0}^{T}\|x(s)\|_{H}^{2} d s<\infty$ a.s. $[P]$, and

$$
x(t)=S(t) x_{0}+\int_{0}^{t} S(t-s) f(s) d s+\int_{0}^{t} S(t-s) g(s) d W(s), \quad \text { a.s. }[P],
$$

for all $0 \leq t \leq T$. 
(The second integral in (2.3) is taken in the sense of Itó. A complete discussion of the construction of the Itó integral can be found in [14].) It is well-known that (2.2) has a unique mild solution $x \in \mathcal{C}([0, T] ; H)$, and if stronger regularity restrictions are imposed on the data, this solution is a strong solution (see $[19,20]$ ).

The following alternative of the Leray-Schauder principle [24] plays a key role in Section 4.

Theorem 2.3: (Schaefer's Fixed Point Theorem [31]) Let $X$ be a Banach space and $\Phi: X \rightarrow X$ a continuous, compact map. Then, either the set $\xi(\Phi)=\{x \in X: \lambda x=\Phi x$, for some $\lambda \geq 1\}$ is unbounded, or $\Phi$ has a fixed point.

We conclude this section with some comments regarding probability measures. We refer the reader to $[9,11]$ for a more detailed discussion.

Let $X$ be an $H$-valued random variable and let $\mathcal{P}(H)$ denote the set of all probability measures on $H$. The probability measure $P$ induced by $X$, denoted $P_{X}$, is defined by $P_{o X}{ }^{-1}: \mathcal{B}(H) \rightarrow[0,1]$, where $\mathcal{B}(H)$ is the Borel class on $H$. A sequence $\left\{P_{n}\right\} \subset \mathcal{P}(H)$ is said to be weakly convergent to $P$ if $\int_{\Omega} f d P_{n} \rightarrow \int_{\Omega} f d P$, for every bounded, continuous function $f: H \rightarrow \mathbb{R}$; in such case, we write $P_{n} \stackrel{w}{\rightarrow} P$. Next, a family $\left\{P_{n}\right\}$ is tight if for each $\epsilon>0$, there exists a compact set $K_{\epsilon}$ such that $P_{n}\left(K_{\epsilon}\right) \geq 1-\epsilon$, for all $n$. Prokhorov [11] established the equivalence of tightness and relative compactness of a family of probability measures. Consequently, the Arzelá-Ascoli Theorem can be used to establish tightness.

Definition 2.4: Let $P \in \mathcal{P}(H)$ and $0 \leq t_{1}<t_{2}<\ldots<t_{k} \leq T$. Define $\pi_{t_{1}, \ldots, t_{k}}$ : $C([0, T] ; H) \rightarrow H^{k}$ by $\pi_{t_{1}, \ldots, t_{k}}(X)=\left(X\left(t_{1}\right), \ldots, X\left(t_{k}\right)\right)$. The probability measures induced by $\pi_{t_{1}, \ldots, t_{k}}$ are the finite dimensional joint distributions of $P$.

Proposition 2.5: ([23], pg. 37) If a sequence $\left\{X_{n}\right\}$ of $H$-valued random variables converges weakly to an $H$-valued random variable $X$ in $L^{2}(\Omega ; H)$, then the sequence of finite dimensional joint distributions corresponding to $\left\{P_{X_{n}}\right\}$ converges weakly to the finite dimensional joint distribution of $P_{X}$.

Finally, the next theorem, in conjunction with Proposition 2.5, is the main tool in establishing a convergence result in Section 5.

Theorem 2.6: Let $\left\{P_{n}\right\} \subset \mathcal{P}(H)$. If the sequence of finite dimensional joint distributions corresponding to $\left\{P_{n}\right\}$ converges weakly to the finite dimensional joint distribution of $P$ and $\left\{P_{n}\right\}$ is relatively compact, then $P_{n} \stackrel{w}{\rightarrow} P$.

\section{Existence Results - Lipschitz Case}

Consider the initial-value problem (1.1) in a real separable Hilbert space $H$ under the following assumptions:

(H1) The linear operator $A: D(A) \subset H \rightarrow H$ generates a $C_{0}$-semi-group $\{S(t): t \geq 0\}$ on $H$,

(H2) $F: \mathcal{C}([0, T] ; H) \rightarrow L^{p}\left(0, T ; L^{2}(\Omega ; H)\right)$ is such that there exists $M_{F}>0$ for which

$$
\|F(x)-F(y)\|_{L^{p}} \leq M_{F}\|x-y\|_{\mathcal{C}}, \quad \text { for all } x, y \in \mathcal{C}([0, T] ; H),
$$

(H3) $G: \mathcal{C}([0, T] ; H) \rightarrow C\left([0, T] ; L^{2}(\Omega ; B L(K ; H))\right)\left(=\mathcal{C}_{B L}\right)$ is such that there exists $M_{G}>0$ for which

$$
\|G(x)-G(y)\|_{\mathcal{C}_{B L}} \leq M_{G}\|x-y\|_{\mathcal{C}}, \quad \text { for all } x, y \in \mathcal{C}([0, T] ; H),
$$


$(\mathrm{H} 4) \quad h: \mathcal{C}([0, T] ; H) \rightarrow L_{0}^{2}(\Omega ; H)$ is such that there exists $M_{h}>0$ for which

$$
\|h(x)-h(y)\|_{L_{0}^{2}} \leq M_{h}\|x-y\|_{\mathcal{C}}, \quad \text { for all } x, y \in \mathcal{C}([0, T] ; H),
$$

(H5) $x_{0} \in L_{0}^{2}(\Omega ; H)$.

Definition 3.1: A function $x \in \mathcal{C}([0, T] ; H)$ is a mild solution of $(1.1)$ on $[0, T]$ if $x$ satisfies Definition 2.2 with (2.3) replaced by

$$
x(t)=S(t)\left(h(x)+x_{0}\right)+\int_{0}^{t} S(t-s) F(x)(s) d s+\int_{0}^{t} \int_{0}^{s} S(s-\tau) G(x)(\tau) d W(\tau) d s, \text { a.s. }[P],
$$

for all $0 \leq t \leq T$. (The Uniform Boundedness Principle and the strong continuity of $S(t)$ together guarantee the existence of a positive constant $M_{S}$ such that $\|S(t)\|_{B L} \leq M_{S}$ for all $0 \leq t \leq T$.) Our first result is:

Theorem 3.2: Assume that (H1) - (H5) hold. Then, (1.1) has a unique mild solution on $[0, T]$, if

$$
M_{S}\left[M_{h}+M_{G} T C_{G}+M_{F} T^{1 / q}\right]<1,
$$

where $1 \leq p, q \leq \infty$ are conjugate indices.

Proof: Define the solution map $\mathcal{J}: \mathcal{C}([0, T] ; H) \rightarrow \mathcal{C}([0, T] ; H)$ by

$$
\begin{gathered}
(\mathcal{J} x)(t)=S(t)\left(h(x)+x_{0}\right)+\int_{0}^{t} S(t-s) F(x)(s) d s \\
+\int_{0}^{t} \int_{0}^{s} S(s-\tau) G(x)(\tau) d W(\tau) d s, \quad 0 \leq t \leq T .
\end{gathered}
$$

The continuity of $\mathcal{J}$ is easily verified. Successive applications of Hölder's inequality yields

$$
\begin{gathered}
{\left[E\left\|\int_{0}^{t} S(t-s) F(x)(s) d s\right\|_{H}^{2}\right]^{\frac{1}{2}} \leq T^{\frac{1}{2}} M_{S}\left[\int_{0}^{T}\|F(x)(s)\|_{L^{2}(\Omega ; H)}^{2} d s\right]^{\frac{1}{2}}} \\
\leq T^{(p-1) / p} M_{S}\|F(x)\|_{L^{p}} .
\end{gathered}
$$

Subsequently, an application of (H2), together with Minkowski's inequality, enables us to continue the string of inequalities in (3.4) to conclude that

$$
\left[E\left\|\int_{0}^{t} S(t-s) F(x)(s) d s\right\|_{H}^{2}\right]^{\frac{1}{2}} \leq T^{\frac{1}{q}} M_{S}\left[M_{F}\|x\|_{\mathcal{C}}+\|F(0)\|_{L^{p}}\right] .
$$

Taking the supremum over $[0, T]$ in (3.5) then implies that $\int_{0}^{t} S(t-s) F(x)(s) d s \in$ $\mathcal{C}([0, T] ; H)$, for any $x \in \mathcal{C}([0, T] ; H)$. Further, for such $x, G(x)(s) \in B L(K ; H)$ and $h(x)+x_{0} \in L_{0}^{2}(\Omega ; H)$ (by (H4) and (H5)). Consequently, one can argue as in [20] to conclude that $\mathcal{J}$ is a well-defined.

Next, we show that $\mathcal{J}$ is a strict contraction. Observe that for $x, y \in \mathcal{C}([0, T] ; H)$, we infer from (3.3) that

$$
(\mathcal{J} x)(t)-(\mathcal{J} y)(t)=S(t)(h(x)-h(y))+\int_{0}^{t} S(t-s)[F(x)(s)-F(y)(s)] d s
$$




$$
+\int_{0}^{t} \int_{0}^{t} S(s-\tau)[G(x)(\tau)-G(y)(\tau)] d W(\tau) d s, \quad 0 \leq t \leq T .
$$

For convenience, let $I_{1}, I_{2}$, and $I_{3}$ represent the first, second, and third terms, respectively, on the right-side of (3.6). Squaring both sides and taking the expectation in (3.6) yields, with the help of Young's inequality,

$$
E\|(\mathcal{J} x)(t)-(\mathcal{J} y)(t)\|_{H}^{2} \leq 4\left[E\left\|I_{1}\right\|_{H}^{2}+E\left\|I_{2}\right\|_{H}^{2}+E\left\|I_{3}\right\|_{H}^{2}\right]
$$

and subsequently,

$$
\|(\mathcal{J} x)(t)-(\mathcal{J} y)(t)\|_{\mathcal{C}} \leq 4\left[\left\|I_{1}\right\|_{\mathcal{C}}+\left\|I_{2}\right\|_{\mathcal{C}}+\left\|I_{3}\right\|_{\mathcal{C}}\right] .
$$

Using reasoning similar to that which led to (3.4), one can show that

$$
\left\|I_{1}\right\|_{\mathcal{C}}+\left\|I_{2}\right\|_{\mathcal{C}} \leq M_{S}\left[M_{h}+M_{F} T^{\frac{1}{q}}\right]\|x-y\|_{\mathcal{C}} .
$$

Also, one can modify the argument of Proposition 1.9 in [20] to conclude that there exists a constant $C_{G}$ (depending only on $p, \operatorname{Tr}(Q)$, and $T$ ) such that

$$
\left\|I_{3}\right\|_{\mathcal{C}} \leq M_{S} M_{G} C_{G} T\|x-y\|_{\mathcal{C}} .
$$

Using (3.8) and (3.9) in (3.7) enables us to conclude that $\mathcal{J}$ is a strict contraction, provided that (3.2) is satisfied and thus, has a unique fixed point which coincides with a mild solution of (1.1). This completes the proof.

Next, we consider the following initial-value problem studied in [16].

$$
\begin{gathered}
x^{\prime}(t)=A x(t)+\int_{0}^{t} C(t, s) g(s, x(s)) d W(s)+\int_{0}^{t} B(t, s) f_{1}(s, x(s)) d s \\
+f_{2}(t, x(t)), \quad 0 \leq t \leq T, \\
x(0)=x_{0},
\end{gathered}
$$

where $\{B(t, s): 0 \leq t \leq s \leq T\} \bigcup\{C(t, s): 0 \leq t \leq s \leq T\} \subset B L(H), g:[0, T] \times H$ $\rightarrow B L(K ; H)$, and $f_{i}:[0, T] \times H \rightarrow H(i=1,2)$ are given mappings satisfying the following conditions:

(H6) $f_{i}:[0, T] \times H \rightarrow H(i=1,2)$ is such that there exists $M_{f_{i}}>0$ for which

$$
\left\|f_{i}(t, x)-f_{i}(t, y)\right\|_{H} \leq M_{f_{i}}\|x-y\|_{H}, \quad \text { for all } t \in[0, T] \text { and } x, y \in H,
$$

(H7) $g:[0, T] \times H \rightarrow B L(K ; H)$ is such that there exists $M_{g}>0$ for which

$$
\|g(t, x)-g(t, y)\|_{B L} \leq M_{g}\|x-y\|_{H}, \quad \text { for all } t \in[0, T] \text { and } x, y \in H .
$$

We recover Theorem 2.1 in [16] as the following corollary of Theorem 3.2.

Corollary 3.3: If (H1), (H4) - (H7), and (3.2) hold, then (3.10) has a unique mild solution on $[0, T]$.

Proof: Define $F: \mathcal{C}([0, T] ; H) \rightarrow L^{1}\left(0, T ; L^{2}(\Omega ; H)\right)$ and $G: \mathcal{C}([0, T] ; H) \rightarrow \mathcal{C}_{B L}$, respectively, by

$$
F(x)(t)=\int_{0}^{t} B(t, s) f_{1}(s, x(s)) d s+f_{2}(t, x(t)), \quad 0 \leq t \leq T,
$$




$$
G(x)(t)=C(t, s) g(t, x(t)), \quad 0 \leq t \leq s \leq T .
$$

The Uniform Boundedness Principle guarantees the existence of positive constants $M_{B}$ and $M_{C}$ such that $\|B(t, s)\|_{B L} \leq M_{B}$ and $\|C(t, s)\|_{B L} \leq M_{C}$, for all $0 \leq t \leq s \leq$ $T$. Standard computations involving properties of expectation and Hölder's inequality imply, with the help of $(\mathrm{H} 6)$, that for all $x, y \in \mathcal{C}([0, T] ; H)$,

$$
\begin{gathered}
\|F(x)-F(y)\|_{L^{1}} \\
\leq 2 \int_{0}^{T}\left[T M_{B}^{2} \int_{0}^{t} E\left\|f_{1}(\tau, x(\tau))-f_{1}(\tau, y(\tau))\right\|_{H}^{2} d \tau+E\left\|f_{2}(s, x(s))-f_{2}(s, y(s))\right\|_{H}^{2}\right]^{\frac{1}{2}} \\
\leq 2 T\left[T M_{B} M_{f_{1}}+M_{f_{2}}\right]\|x-y\|_{\mathcal{C}} .
\end{gathered}
$$

Similarly, (H7) enables us to infer that for all $x, y \in \mathcal{C}([0, T] ; H)$,

$$
\|G(x)-G(y)\|_{\mathcal{C}_{B L}} \leq M_{C} M_{g}\|x-y\|_{\mathcal{C}} .
$$

Thus, if we let $M_{F}=2 T\left[T M_{B} M_{f_{1}}+M_{f_{2}}\right]$ in (H2) and $M_{G}=M_{C} M_{g}$ in (H3), and take $h=0$, we can conclude from Theorem 3.2 that (3.10) has a unique mild solution on $[0, T]$.

\section{Remark 3.4:}

(i) We also recover Theorem 3.3 in [22] as a corollary to Theorem 3.2 if we replace $F$ and $G$ in (3.11), respectively, by $F(x)(t)=f(t)$ and $G(x)(t)=C(t-s) x(t)$, for all $0 \leq t \leq s \leq T$, where $C$ is a convolution-type kernel satisfying Assumptions 3.2 on page 361 in [22]. The result then follows from Corollary 3.3.

(ii) A result analogous to Corollary 3.3 regarding a delay version of (3.10) (obtained by replacing $g(s, x(s))$ by $g(s, x(s), x(\sigma(s)))$, where $\sigma:[0, T] \rightarrow[0, T]$ is a continuous, nondecreasing function) can be established by making slight modifications to the above argument. A related delay equation is discussed in [7] using compactness methods.

We conclude this section with a comment on a special case of (3.10), namely where $x_{0}=0$ and $h$ is given by

$$
h(x)=x(T), \quad \text { for all } x \in \mathcal{C}([0, T] ; H) .
$$

Clearly, $h$, as given by (3.14), satisfies (H7) with $M_{g}=1$. Since $M_{S} \geq 1$, condition (3.2) does not hold for such $h$. To incorporate (3.14) into our theory, we consider that the functions $f_{i}$ and $g$ are defined instead on $\mathcal{C}((0, \infty) ; H)$ and satisfy $(\mathrm{H} 6)$ and $(\mathrm{H} 7)$, respectively, with $[0, T]$ replaced by $[0, \infty)$. Also, we take $B$ and $C$ to be convolution kernels in $L^{1}(0, \infty)$ of the type described in Remark 3.4(i). And finally, we assume that $A$ generates a semi-group $\{S(t): t \geq 0\}$ on $H$ such that

(H8) There exist $M_{S} \geq 1$ and $\omega>0$ such that $\|S(t)\|_{B L} \leq M_{S} e^{-\omega t}$, for all $t \geq 0$.

For conditions that ensure that (H8) holds, see [30], pg. 116. Using an approach similar to the one employed in [25], we can now prove that the following initial-value problem has a unique mild solution, provided $T$ is sufficiently large.

$$
x^{\prime}(t)=A x(t)+\int_{0}^{t} C(t-s) g(s, x(s)) d W(s)+\int_{0}^{t} B(t-s) f_{1}(s, x(s)) d s
$$




$$
\begin{gathered}
+f_{2}(t, x(t)), \quad 0 \leq t \leq T, \\
x(0)=x(T) .
\end{gathered}
$$

Theorem 3.5: Suppose (H1) and (H8) hold, and that $f_{i}, g, B$, and $C$ are as described above. If also

(H9) $M_{S} \exp \left[-\omega T+M_{S}\left(M_{f_{1}}\|B\|_{L^{1}(0, \infty)}+M_{f_{2}}+M_{g}\|C\|_{L^{1}(0, \infty)}\right)\right]<1$, then (3.15) has a unique mild solution on $[0, T]$.

Proof: Arguing as in [22], it follows that for each fixed $T>0$ and each $y \in L_{0}^{2}(\Omega ; H)$, the initial-value problem (3.15) (with $y$ in place of $x(T)$ ) has a unique mild solution $x_{y}$ on $[0, T]$ given by

$$
\begin{gathered}
x_{y}(t)=S(t) y+\int_{0}^{t} \int_{0}^{s} S(s-\tau) B(s-u) f_{1}(\tau, x(\tau)) d \tau d s+\int_{0}^{t} S(t-s) f_{2}(s, x(s)) d s \\
+\int_{0}^{t} \int_{0}^{s} S(s-\tau) C(s-\tau) g(\tau, x(\tau)) d W(\tau) d s, \quad 0 \leq t \leq T .
\end{gathered}
$$

On account of (H8), and the assumptions imposed on $f_{i}, g, B$, and $C,(3.16)$ yields

$$
\begin{aligned}
\left\|x_{y}(t)-x_{z}(t)\right\|_{H} \leq & M_{S} e^{-\omega t}\|y-z\|_{H}+M_{S}\left(M_{f_{1}}\|B\|_{L^{1}(0, \infty)}+M_{f_{2}}+M_{g}\|C\|_{L^{1}(0, \infty)}\right) \\
& \cdot \int_{0}^{t} e^{-\omega(t-s)}\left\|x_{y}(s)-x_{z}(s)\right\|_{H} d s, \quad 0 \leq t \leq T .
\end{aligned}
$$

Now, using a Gronwall-type inequality in (3.17) (cf. [25], Lemma 4.2), we arrive at

$\left\|x_{y}(T)-x_{z}(T)\right\|_{H} \leq M_{S} \exp \left[-\omega T+M_{S}\left(M_{f_{1}}\|B\|_{L^{1}(0, \infty)}+M_{f_{2}}+M_{g}\|C\|_{L^{1}(0, \infty)}\right)\right] \cdot\|y-z\|_{H}$,

for all $y, z \in L_{0}^{2}(\Omega ; H)$, and subsequently,

$$
\begin{gathered}
\left\|x_{y}(T)-x_{z}(T)\right\|_{L^{2}(\Omega ; H)} \leq M_{S} \exp \left[-\omega T+M_{S}\left(M_{f_{1}}\|B\|_{L^{1}(0, \infty)}+M_{f_{2}}+M_{g}\|C\|_{L^{1}(0, \infty)}\right)\right] \\
\cdot\|y-z\|_{L_{0}^{2}} .
\end{gathered}
$$

Define $Q_{T}: L^{2}(\Omega ; H) \rightarrow L^{2}(\Omega ; H)$ by $Q_{T}(y)=u_{y}(T)$. Observe that (3.18) and (H9) imply that $Q_{T}$ is a strict contraction on $L^{2}(\Omega ; H)$, for sufficiently large $T$. Thus, for $T$ chosen such that (H9) is satisfied, $Q_{T}$ has a unique fixed point $y_{0}$. The corresponding function $u=u_{y_{0}}$ is the desired mild solution of (3.15).

\section{Existence Results - Compactness Case}

We now develop existence results for (1.1) in which the Lipschitz conditions on $F, G$, and $h$ are replaced by sublinear growth conditions. This is done at the expense of a compactness restriction on the semi-group. Precisely, we use the following assumptions instead:

(H10) $A$ generates a compact $C_{0}$-semi-group $\{S(t): t \geq 0\}$ on $H$,

(H11) $F: \mathcal{C}([0, T] ; H) \rightarrow L^{p}\left(0, T ; L^{2}(\Omega ; H)\right)$ is a continuous map for which there exists positive constants $c_{1}$ and $c_{2}$ such that $\|F(x)\|_{L^{p}} \leq c_{1}\|x\|_{\mathcal{C}}+c_{2}$, for all $x \in \mathcal{C}([0, T] ; H)$, 
(H12) $G: \mathcal{C}([0, T] ; H) \rightarrow \mathcal{C}_{B L}$ is a continuous map for which there exists $d_{1}>0$ and $d_{2} \in L^{2}\left(0, T ; \mathbb{R}^{+}\right)$such that $\|G(x)\|_{\mathcal{C}_{B L}} \leq d_{1}\|x\|_{\mathcal{C}}+d_{2}(\cdot)$, for all $x \in \mathcal{C}([0, T] ; H)$,

$(\mathrm{H} 13) h: \mathcal{C}([0, T] ; H) \rightarrow L_{0}^{2}(\Omega ; H)$ is a continuous, compact map for which there exists positive constants $e_{1}$ and $e_{2}$ such that $\|h(x)\|_{L_{0}^{2}} \leq e_{1}\|x\|_{\mathcal{C}}+e_{2}$, for all $x \in$ $\mathcal{C}([0, T] ; H)$.

We begin by establishing certain compactness properties of the mappings $\Phi_{1}$ : $L^{p}\left(0, T ; L^{2}(\Omega, H)\right) \rightarrow \mathcal{C}([0, T] ; H)$ and $\Phi_{2}: \mathcal{C}_{B L} \rightarrow \mathcal{C}([0, T] ; H)$ defined, respectively, by

$$
\begin{gathered}
\Phi_{1}(v)(t)=\int_{0}^{t} S(t-s) v(s) d s, \quad 0 \leq t \leq T, \\
\Phi_{2}(v)(t)=\int_{0}^{t} \int_{0}^{s} S(s-\tau) v(\tau) d W(\tau) d s, \quad 0 \leq s \leq t \leq T .
\end{gathered}
$$

The well-definedness of these two mappings follows from an application of Lebesgue's Dominated Convergence Theorem.

Lemma 4.1: Assume that $\{S(t): 0 \leq t \leq T\}$ is a compact semi-group on $H$. Then,

(i) $\Phi_{1}$ maps uniformly integrable sets in $L^{1}\left(0, T ; L^{2}(\Omega, H)\right)$ into precompact subsets of $\mathcal{C}([0, T] ; H)$. Further, $\Phi_{1}$ is a compact map from $L^{p}\left(0, T ; L^{2}(\Omega ; H)\right)$ into $\mathcal{C}([0, T] ; H)$, for $p>1$,

(ii) $\Phi_{2}$ is a compact map from $\mathcal{C}_{B L}$ into $\mathcal{C}([0, T] ; H)$.

Proof: Part (i) is essentially a stochastic analog of Lemma 3.1 in [3] (where $S(t)$ plays the role of the resolvent operator) and its proof follows similarly by making the natural modifications. We shall only sketch the proof of (ii).

Let $K_{r}=\left\{v \in \mathcal{C}_{B L}:\|v\|_{\mathcal{C}_{B L}} \leq r\right\}$. We shall show that $\Phi_{2}\left(K_{r}\right)$ is equicontinuous at each $t \in[0, T]$ and $\Phi_{2}\left(K_{r}\right)(t)$ is precompact in $L^{2}(\Omega ; H)$, for each $t \in[0, T]$. To this end, observe that for $0<t_{1} \leq t_{2} \leq T$ and $v \in K_{r}$, we have

$$
\begin{gathered}
\left\|\Phi_{2}(v)\left(t_{2}\right)-\Phi_{2}(v)\left(t_{1}\right)\right\|_{L^{2}(\Omega ; H)} \leq\left[T E \int_{t_{1}}^{t_{2}} \int_{0}^{s}\|S(s-\tau) v(\tau)\|_{B L}^{2} d \tau d s\right]^{\frac{1}{2}} \\
\leq M_{S} T^{1 / 2}\left[\int_{t_{1}}^{t_{2}} \int_{0}^{s}\|v\|_{\mathcal{C}_{B L}}^{2} d \tau d s\right]^{\frac{1}{2}} \\
\leq M_{S} T\|v\|_{\mathcal{C}_{B L}}\left(t_{2}-t_{1}\right)^{1 / 2} .
\end{gathered}
$$

Observe that the right-side of (4.3) tends to zero as $t_{2} \rightarrow t_{1}$, uniformly for $v \in K_{r}$. A similar argument works for $t=0$, thereby verifying the equicontinuity.

Next, note that the precompactness of $\Phi_{2}\left(K_{r}\right)(0)=\{0\}$ is trivial. Let $0<t \leq T$, $0<\epsilon<t$, and define by $\Phi_{2}^{\epsilon}: \mathcal{C}_{B L} \rightarrow \mathcal{C}([0, T] ; H)$ by

$$
\Phi_{2}^{\epsilon}(v)(t)=\int_{0}^{t-\epsilon} \int_{0}^{s} S(s-\tau) v(\tau) d W(\tau) d s, \quad 0 \leq t \leq s \leq T .
$$

We claim that $K_{2}(\epsilon ; t)=\left\{\Phi_{2}^{\epsilon}(v)(t): v \in K_{r}\right\}$ is precompact in $L^{2}(\Omega ; H)$. Indeed, observe that

$$
\left\|\Phi_{2}(v)(t)-\Phi_{2}^{\epsilon}(v)(t)\right\|_{L^{2}(\Omega ; H)}
$$




$$
\leq M_{S} T^{1 / 2}\left[\int_{t-\epsilon}^{t} \int_{0}^{s}\|v\|_{\mathcal{C}_{B L}}^{2} d \tau d s\right]^{\frac{1}{2}} \leq M_{S} \operatorname{Tr} \epsilon^{1 / 2}, 0<\epsilon<t .
$$

Since the right-side of (4.4) can be made arbitrarily small, uniformly for $v \in K_{r}$, we conclude that $\Phi_{2}\left(K_{r}\right)(t)$ is totally bounded. This, combined with the work above, yields the precompactness, and the proof is complete.

Theorem 4.2: Assume that (H5) and (H10) - (H13) are satisfied. Then, (1.1) has at least one mild solution on $[0, T]$ provided that

(H14) $2 M_{S}\left[e_{1}+\sqrt{2} T^{3 / 2} d_{1}+T^{1 / q} c_{1}\right]<1$.

Proof: We use Schaefer's theorem to prove that $\mathcal{J}$ (as defined in (3.3)) has a fixed point.

The well-definedness of $\mathcal{J}$ under (H10) - (H13) can be established using reasoning similar to that employed in the proof of Theorem 3.2. To verify the continuity of $\mathcal{J}$, let $\left\{v_{n}\right\}_{n=1}^{\infty}$ be a sequence in $\mathcal{C}([0, T] ; H)$ such that $v_{n} \rightarrow v$ as $n \rightarrow \infty$. Standard computations yield

$$
\begin{gathered}
\left\|\mathcal{J}\left(v_{n}\right)-\mathcal{J}(v)\right\|_{\mathcal{C}} \leq 2 M_{S}\left[\left\|h\left(v_{n}\right)-h(v)\right\|_{L_{0}^{2}}\right. \\
+\left(E\left(\int_{0}^{T}\left\|F\left(v_{n}\right)(s)-F(v)(s)\right\|_{H} d s\right)^{2}\right)^{1 / 2} \\
\left.+\left(E\left(\int_{0}^{T} \int_{0}^{T}\left\|G\left(v_{n}\right)(\tau)-G(v)(\tau)\right\|_{B L} d W(\tau) d s\right)^{2}\right)^{1 / 2}\right] \\
\leq 2 M_{S}\left[\left\|h\left(v_{n}\right)-h(v)\right\|_{L_{0}^{2}}+T^{1 / q}\left\|F\left(v_{n}\right)-F(v)\right\|_{L^{p}}+T^{3 / 2}\left\|G\left(v_{n}\right)-G(v)\right\|_{\mathcal{C}_{B L}}\right]
\end{gathered}
$$

The continuity of $F, G$, and $h$ ensure that the right-side of (4.5) goes to zero as $n \rightarrow \infty$, thereby verifying the continuity of $\mathcal{J}$.

Next, we show that the set $\xi(\mathcal{J})$, as defined in Theorem 2.3 with $\mathcal{C}([0, T] ; H)$ in place of $X$, is bounded. Let $v \in \xi(\mathcal{J})$ and observe that the Hölder and Young inequalities (with (H12)) yield

$$
T^{1 / 2}\left(E \int_{0}^{T} \int_{0}^{T}\|G(v)(\tau)\|_{B L}^{2} d \tau d s\right)^{1 / 2} \leq \sqrt{2} T^{3 / 2} d_{1}\|v\|_{\mathcal{C}}+\sqrt{2} T^{1 / 2}\left\|d_{2}\right\|_{L^{2}(0, T)} .
$$

Also, arguing as in (3.4), we obtain (with the help of (H14))

$$
T^{1 / 2}\left(E \int_{0}^{T}\|F(v)(s)\|_{H}^{2} d s\right)^{1 / 2} \leq T^{1 / q}\left(c_{1}\|v\|_{\mathcal{C}}+c_{2}\right) .
$$

Hence, (4.6) and (4.7), in conjunction with (H13), enable us to conclude that for all $v \in \xi(\mathcal{J})$ and $0 \leq t \leq T$, we have

$$
\lambda\|v\|_{\mathcal{C}} \leq 2 M_{S}\left[e_{1}\|v\|_{\mathcal{C}}+e_{2}+\left\|x_{0}\right\|_{L_{0}^{2}}+\sqrt{2} T^{3 / 2} d_{1}\|v\|_{\mathcal{C}}+\sqrt{2} T^{1 / 2}\left\|d_{2}\right\|_{L^{2}(0, T)}\right.
$$




$$
\left.+T^{1 / q}\left(c_{1}\|v\|_{\mathcal{C}}+c_{2}\right)\right] .
$$

Taking into account that $\lambda \geq 1$ and (H14), we conclude from (4.7) that $\|v\|_{\mathcal{C}} \leq \eta$, where $\eta$ is a constant independent of $v$ and $\lambda$. So, $\xi(\mathcal{J})$ is bounded.

To apply Schaefer's theorem, we must finally show that $\mathcal{J}$ is compact. To this end, let $r>0$ and define $K_{r}=\left\{v \in \mathcal{C}([0, T] ; H):\|v\|_{\mathcal{C}} \leq r\right\}$. Using the notation of (4.1) and (4.2), we can express (3.3) as

$$
\mathcal{J}(v)=S(\cdot)\left(h(v)+x_{0}\right)+\Phi_{1}(F(v)(\cdot))+\Phi_{2}(G(v)(\cdot)), v \in \mathcal{C}([0, T] ; H) .
$$

We shall prove that $\mathcal{J}\left(K_{r}\right)$ is precompact in $\mathcal{C}([0, T] ; H)$. First, the facts that $\{F(v)$ : $\left.v \in K_{r}\right\}$ and $\left\{G(v): v \in K_{r}\right\}$ are bounded subsets of $L^{p}\left(0, T ; L^{2}(\Omega ; H)\right)$ and $\mathcal{C}_{B L}$, respectively (cf. (H11) and (H12)), it follows from Lemma 4.1 that the set $\left\{\Phi_{1}(F(v))+\right.$ $\left.\Phi_{2}(G(v)): v \in K_{r}\right\}$ is precompact in $\mathcal{C}([0, T] ; H)$. It remains to establish the precompactness of $\left\{S(\cdot)\left(h(v)+x_{0}\right): v \in K_{r}\right\}$. Since $\left\{S(\cdot)\left(x_{0}\right): v \in K_{r}\right\}=\left\{S(\cdot)\left(x_{0}\right)\right\}$ is trivially precompact, we need only focus on $\left\{S(\cdot)(h(v)): v \in K_{r}\right\}$. By (H13), the set $L=\left\{h(v): v \in K_{r}\right\}$ is precompact in $L_{0}^{2}(\Omega ; H)$. Let $\tilde{L}=S(\cdot) L(\subset \mathcal{C}([0, T] ; H))$ and $\epsilon>$ 0 . The precompactness of $L$ in $L_{0}^{2}(\Omega ; H)$ guarantees the existence of $\left\{x_{1}, \ldots, x_{n}\right\} \subset L$ such that $L \subset \bigcup_{i=1}^{n} B\left(x_{i}, \epsilon / M_{S}\right)$, where $B\left(x_{i}, \epsilon / M_{S}\right)$ is the ball in $L_{0}^{2}(\Omega ; H)$ with radius $\epsilon / M_{S}$ and center $x_{i}$. Then, $\tilde{L} \subset \bigcup_{i=1}^{n} S(\cdot) B\left(x_{i}, \epsilon / M_{S}\right)$. Let $\tilde{x}_{i}=S(\cdot) x_{i} \in \mathcal{C}([0, T] ; H)$ and $\tilde{B}_{i}=\left\{y \in \mathcal{C}([0, T] ; H):\left\|y-\tilde{x}_{i}\right\|_{\mathcal{C}}<\epsilon\right\}$. For $z \in \tilde{L}$, there exists $\Psi \in L$ such that $z \in S(\cdot) \Psi$. Since $\Psi \in L$, there is an $i \in\{1, \ldots, n\}$ such that $\left\|\Psi-x_{i}\right\|_{L_{0}^{2}}<\epsilon / M_{S}$. Observe that $\left\|z-\tilde{x}_{i}\right\|_{\mathcal{C}}=\left\|S(\cdot) \Psi-S(\cdot) x_{i}\right\|_{\mathcal{C}} \leq M_{S}\left\|\xi-x_{i}\right\|_{L_{0}^{2}}<\epsilon$. It then follows that $\tilde{L} \subset \bigcup_{i=1}^{n} \tilde{B}_{i}$ and hence, $\tilde{L}$ is totally bounded. Thus, $\tilde{L}$ is precompact in $\mathcal{C}([0, T] ; H)$. Hence, Schaefer's theorem implies that $\mathcal{J}$ has at least one fixed point $x \in \mathcal{C}([0, T] ; H)$ which is a mild solution to (1.1).

Next, we state a corollary regarding (3.10) under the following assumptions on $f_{i}$ and $g$ :

(H15) $f_{i}:[0, T] \times H \rightarrow H(i=1,2)$ satisfies

(i) $f_{i}(t, \cdot): H \rightarrow H$ is continuous, for almost all $t \in[0, T]$,

(ii) $f_{i}(\cdot, x):[0, T] \rightarrow H$ is strongly $\mathcal{F}_{t}$-measurable, for all $x \in H$,

(iii) there exist positive constants $a_{i, 1}$ and $a_{i, 2}$ such that $\left\|f_{i}(t, x)\right\|_{H} \leq a_{i, 1}\|x\|_{H}+$ $a_{i, 2}$ for almost all $t \in[0, T]$ and for all $x \in H$,

(H16) $g:[0, T] \times H \rightarrow B L(K ; H)$ satisfies

(i) $g(t, \cdot): H \rightarrow B L(K ; H)$ is continuous, for almost all $t \in[0, T]$,

(ii) $g(\cdot, x):[0, T] \rightarrow B L(K ; H)$ is strongly $\mathcal{F}_{t}$-measurable, for all $x \in H$,

(iii) there exist positive constants $b_{1}$ and $b_{2}$ such that $\|g(t, x)\|_{B L} \leq b_{1}\|x\|_{H}+b_{2}$ for almost all $t \in[0, T]$ and for all $x \in H$.

Corollary 4.3: If (H5), (H10), and (H13)-(H16) are satisfied, then (3.10) has at least one mild solution on $[0, T]$.

Proof: An argument similar to the one used in [34], (Chapter 26, pg. 561) can be used to show that (H15) and (H16) guarantee that the mappings $F: \mathcal{C}([0, T] ; H) \rightarrow$ $L^{1}\left(0, T ; L^{2}(\Omega ; H)\right)$ and $G: \mathcal{C}([0, T] ; H) \rightarrow \mathcal{C}_{B L}$ defined in (3.11) are well-defined and continuous. Further, routine calculations show that $F$ and $G$ satisfy (H11) and (H12), 
respectively, with $c_{1}=2 T\left(a_{1,1} M_{B} T^{3 / 2}+a_{2,1}\right), c_{2}=2 T\left(a_{1,2} M_{B} T^{3 / 2}+a_{2,2}\right), d_{1}=$ $2 M_{C} b_{1} T$, and $d_{2}=2 M_{C} b_{2} T$. Consequently, (3.10) has at least one mild solution by Theorem 4.2.

We can formulate a stronger version of Corollary 4.3 by replacing assumption (H15) and (H16), respectively, by

(H17) $f_{i}:[0, T] \times H \rightarrow H(i=1,2)$ satisfies (H15) (i) and (ii), as well as

(i) For each $k \in \mathbb{N}$, there exists $g_{i, k} \in L^{1}\left(0, T ; \mathbb{R}^{+}\right)$such that for almost all $t \in(0, T), \sup _{\|x\|_{H} \leq k} E\left\|f_{i}(t, x)\right\|_{H}^{2} \leq g_{i, k}(t)$,

(ii) $\underline{\lim }_{k \rightarrow \infty} k^{-2} \int_{0}^{T} g_{i, k}(s) d s=\alpha_{i}<\infty$,

(H18) $g:[0, T] \times H \rightarrow B L(K ; H)$ satisfies (H16) (i) and (ii), as well as

(i) For each $k \in \mathbb{N}$ there exists $j_{k} \in L^{1}\left(0, T ; \mathbb{R}^{+}\right)$such that for almost all $t \in(0, T), \sup _{\|x\|_{H} \leq k} E\|g(t, x)\|_{B L}^{2} \leq j_{k}(t)$,

(ii) $\underline{\lim }_{k \rightarrow \infty} k^{-2} \int_{0}^{T} j_{k}(s) d s=\beta<\infty$.

Comparable conditions appear in $[7,33]$.

Proposition 4.4: Assume that (H5), (H10), (H13), (H17), and (H18) are satisfied. If, in addition,

(H19) $4 M_{S}\left[e_{1}+T^{1 / 2}\left(\alpha_{2}^{1 / 2}+M_{B} T^{3} \alpha_{1}^{1 / 2}+M_{C} T^{2} \beta^{1 / 2}\right)\right]<1$, then (3.10) has at least one mild solution on $[0, T]$.

Proof: We use Schauder's fixed-point theorem [24] to argue that $\mathcal{J}$ (as defined in (3.3) with $F$ and $G$ given by (3.11) has a fixed point. The continuity and compactness follow by making slight changes to the proof of Theorem 4.1. For $n \in \mathbb{N}$, let $B_{n}=$ $\left\{x \in \mathcal{C}([0, T] ; H):\|x\|_{\mathcal{C}} \leq n\right\}$. It remains to show that there exists an $n \in \mathbb{N}$ such that $\mathcal{J}\left(B_{n}\right) \subset B_{n}$.

Suppose, by way of contradiction, that for each $k \in \mathbb{N}$, there exists $u_{k} \in B_{k}$ such that $\mathcal{J}\left(u_{k}\right) \notin B_{k}$. Then,

$$
1 \leq \underline{\lim }_{k \rightarrow \infty} k^{-1}\left\|\mathcal{J}\left(u_{k}\right)\right\|_{\mathcal{C}}
$$

Observe that

$$
\begin{gathered}
\left\|\mathcal{J}\left(u_{k}\right)\right\|_{\mathcal{C}} \\
\leq 4 M_{S}\left[\left\|h\left(u_{k}\right)\right\|_{L_{0}^{2}}+\left\|x_{0}\right\|_{L_{0}^{2}}+T^{1 / 2}\left(T^{1 / 2} M_{B}\left(\int_{0}^{T} \int_{0}^{T} E\left\|f_{1}\left(\tau, u_{k}(\tau)\right)\right\|_{H}^{2} d \tau d s\right)^{1 / 2}\right.\right. \\
\left.\left.+\left(\int_{0}^{T} E\left\|f_{2}\left(s, u_{k}(s)\right)\right\|_{H}^{2} d s\right)^{1 / 2}+T^{1 / 2} M_{C}\left(\int_{0}^{T} \int_{0}^{T} E\left\|g\left(\tau, u_{k}(\tau)\right)\right\|_{B L}^{2} d \tau d s\right)^{1 / 2}\right)\right] .
\end{gathered}
$$

Note that for each $k \in \mathbb{N}, u_{k} \in B_{k}$ and hence, $\left\|u_{k}(s)\right\|_{H} \leq k$, for all $0 \leq s \leq T$. So, by (H17) and (H18), there exist $g_{i, k}(i=1,2), j_{k} \in L^{1}\left(0, T ; \mathbb{R}^{+}\right)$such that for almost all $0 \leq s \leq T$

$$
\begin{gathered}
E\left\|f_{i}\left(s, u_{k}(s)\right)\right\|_{H}^{2} \leq g_{i, k}(s), \quad(i=1,2), \\
E\left\|g\left(s, u_{k}(s)\right)\right\|_{B L}^{2} \leq j_{k}(s) .
\end{gathered}
$$


Using (4.12) in (4.11) yields (with the help of (H13))

$$
\begin{gathered}
\left\|\mathcal{J}\left(u_{k}\right)\right\|_{\mathcal{C}} \leq 4 M_{S}\left[\left[e_{1}\left\|u_{k}\right\|_{\mathcal{C}}+e_{2}+\left\|x_{0}\right\|_{L_{0}^{2}}\right]+T^{1 / 2}\left(\int_{0}^{T} g_{2, k}(s) d s\right)^{1 / 2}\right. \\
\left.+M_{B} T^{3 / 2}\left(\int_{0}^{T} g_{1, k}(s) d s\right)^{1 / 2}+M_{C} T^{1 / 2}\left(\int_{0}^{T} j_{k}(s) d s\right)^{1 / 2}\right] .
\end{gathered}
$$

and subsequently,

$$
\begin{gathered}
\underline{\lim }_{k \rightarrow \infty} k^{-1}\left\|\mathcal{J}\left(u_{k}\right)\right\|_{\mathcal{C}} \leq 4 M_{S} \underline{\lim }_{k \rightarrow \infty}\left[e_{1} k^{-1}\left\|u_{k}\right\|_{\mathcal{C}}+\left(e_{2}+\left\|x_{0}\right\|_{L_{0}^{2}}\right) k^{-1}\right. \\
+4 M_{S} T^{1 / 2}\left(k^{-2} \int_{0}^{T} g_{2, k}(s) d s\right)^{1 / 2} \\
\left.+M_{B} T^{3 / 2}\left(k^{-2} \int_{0}^{T} g_{1, k}(s) d s\right)^{1 / 2}+4 M_{S} M_{C} T\left(k^{-2} \int_{0}^{T} j_{k}(s) d s\right)^{1 / 2}\right] \\
\leq 4 M_{S}\left[e_{1}+T^{1 / 2}\left(\alpha_{2}^{1 / 2}+M_{B} T^{3} \alpha_{1}^{1 / 2}+M_{C} T^{2} \beta^{1 / 2}\right)\right] \\
<1(\text { by }(\mathrm{H} 19)),
\end{gathered}
$$

contradicting (4.10). Consequently, there is an $n_{0} \in \mathbb{N}$ such that $\mathcal{J}\left(B_{n_{0}}\right) \subset B_{n_{0}}$. Thus, Schauder's fixed point theorem guarantees the existence of $x \in B_{n_{0}}$ such that $\mathcal{J}(x)=x$, which is the mild solution that we seek.

Remark: An inspection of the proof shows that (H13) can be weakened slightly in that instead of imposing the sublinear growth restriction on $h$, we need only assume that $\lim _{\|x\|_{\mathcal{C}} \rightarrow \infty}\|h(x)\|_{L_{0}^{2}} /\|x\|_{\mathcal{C}}=\zeta<\infty$.

\section{Convergence Results}

Throughout this section we assume that $A, F, G$, and $h$ satisfy (H1) - (H4) and that (3.2) holds. For each $n \in \mathbb{N}$, consider a linear operator $A_{n}: D\left(A_{n}\right)(=D(A)) \rightarrow H$ and mappings $F_{n}: \mathcal{C}([0, T] ; H) \rightarrow L^{p}\left(0, T ; L^{2}(\Omega ; H)\right), G_{n}: \mathcal{C}([0, T] ; H) \rightarrow \mathcal{C}_{B L}$, and $h_{n}: \mathcal{C}([0, T] ; H) \rightarrow L_{0}^{2}(\Omega ; H)$ satisfying the following conditions:

(H20) $A_{n}$ generates a $C_{0}$-semi-group $\left\{S_{n}(t): t \geq 0\right\}$ such that $\left\|S_{n}(t)\right\|_{B L} \leq M_{S} e^{\alpha t}$, for some $\alpha>0$ (independent of $n$ ), for each $n \in \mathbb{N}$, and $A_{n} x \rightarrow A x$ strongly as $n \rightarrow \infty$, for each $x \in D(A)$,

(i) $\left\|F_{n}(x)-F_{n}(y)\right\|_{L^{p}} \leq M_{F}\|x-y\|_{\mathcal{C}}$, for all $x, y \in \mathcal{C}([0, T] ; H)$,

(ii) $F_{n}(x) \stackrel{L^{p}}{\rightarrow} F(x)$ as $n \rightarrow \infty$, for all $x \in \mathcal{C}([0, T] ; H)$,

(i) $\left\|G_{n}(x)-G_{n}(y)\right\|_{\mathcal{C}_{B L}} \leq M_{G}\|x-y\|_{\mathcal{C}}$, for all $x, y \in \mathcal{C}([0, T] ; H)$,

(ii) $G_{n}(x) \stackrel{\mathcal{C}_{B L}}{\longrightarrow} G(x)$ as $n \rightarrow \infty$, for all $x \in \mathcal{C}([0, T] ; H)$,

(i) $\left\|h_{n}(x)-h_{n}(y)\right\|_{L_{0}^{2}} \leq M_{h}\|x-y\|_{\mathcal{C}}$, for all $x, y \in \mathcal{C}([0, T] ; H)$, 
(ii) $h_{n}(x) \stackrel{L_{0}^{2}}{\rightarrow} h(x)$ as $n \rightarrow \infty$, for all $x \in \mathcal{C}([0, T] ; H)$.

(Here, the constants $M_{S}, M_{F}, M_{G}$, and $M_{h}$ are the same ones appearing in (H1)-(H4) and so, are independent of $n$.)

Let $x$ be the mild solution to (1.1) as guaranteed by Theorem 3.2. By virtue of (H6), (H20), (H21)(i), (H22)(i), and (H23)(i), Theorem 3.2 implies that, for each $n \in \mathbb{N}$, the problem

$$
\begin{gathered}
x_{n}^{\prime}(t)=A_{n} x_{n}(t)+F_{n}\left(x_{n}\right)(t)+\int_{0}^{t} G_{n}\left(x_{n}\right)(s) d W(s), 0 \leq t \leq T, \\
x_{n}(0)=h_{n}\left(x_{n}\right)+x_{0},
\end{gathered}
$$

has a unique mild solution $x_{n} \in \mathcal{C}([0, T] ; H)$.

Consider the following initial-value problem:

$$
\begin{gathered}
y_{n}^{\prime}(t)=A_{n} y_{n}(t)+F_{n}(x)(t)+\int_{0}^{t} G_{n}(x)(s) d W(s), 0 \leq t \leq T, \\
y_{n}(0)=h_{n}(x)+x_{0} .
\end{gathered}
$$

Since $h_{n}(x)+x_{0}$ is a fixed element of $L_{0}^{2}(\Omega ; H)$, a standard argument (see Ch. 7 in [14]) guarantees the existence of a unique mild solution $y_{n}$ of (5.2). We need the following lemma.

Lemma 5.1: If (H20)-(H23) hold, then $y_{n} \stackrel{\mathcal{C}}{\rightarrow} x$ as $n \rightarrow \infty$.

Proof: Using (H20) in conjunction with Theorem 4.1 in [19], pg. 46, we infer that $S_{n}(t) z \rightarrow S(t) z$ strongly as $n \rightarrow \infty$, for all $z \in H$, uniformly in $t \in[0, T]$. Observe that

$$
\begin{gathered}
\left\|y_{n}(t)-x(t)\right\|_{H} \leq\left\|S_{n}(t)\left(h_{n}(x)-h(x)\right)+\left(S_{n}(t)-S(t)\right) h(x)\right\|_{H} \\
+\int_{0}^{t}\left\|S_{n}(t-s)\left(F_{n}(x)(s)-F(x)(s)\right)\right\|_{H} d s+\int_{0}^{t}\left\|\left(S_{n}(t-s)-S(t-s)\right) F(x)(s)\right\|_{H} d s \\
+\left\|\int_{0}^{t} \int_{0}^{s}\left[\left(S_{n}(s-\tau)\left(G_{n}(x)(\tau)-G(x)(\tau)\right)\right)+\left(S_{n}(s-\tau)-S(s-\tau)\right) G(x)(\tau)\right] d W(\tau) d s\right\|_{H} .
\end{gathered}
$$

A standard argument invoking the Trotter-Kato Theorem [30] can be used, invoking (H21)(ii)-(H23)(ii), to complete the proof.

We now state the first of our two main convergence results. A comparable theorem for a nonlinear deterministic evolution equation is discussed in [2].

Theorem 5.2: Assume that $(H 1)-(H 6),(3.2)$, and $(H 20)-(H 23)$ are satisfied. Then, $x_{n} \stackrel{\mathcal{C}}{\rightarrow} x$, provided $8 \overline{M_{S}}\left[M_{h}+T^{1 / q} M_{F}+T^{5 / 2} M_{G}\right]<1$, where $\overline{M_{S}}=M_{S} e^{\alpha T}$.

Proof: Let $y_{n}$ be the mild solution of (5.2). Observe that

$$
\begin{gathered}
\left\|x_{n}(t)-x(t)\right\|_{H}^{2} \leq 4\left[\left\|x_{n}(t)-y_{n}(t)\right\|_{H}^{2}+\left\|y_{n}(t)-x(t)\right\|_{H}^{2}\right] \\
\quad \leq 4\left\{1 6 \left[\left\|S_{n}(t)\left(h_{n}\left(x_{n}\right)+x_{0}-h_{n}(x)-x_{0}\right)\right\|_{H}^{2}\right.\right. \\
+\left(\int_{0}^{T}\left\|S_{n}(T-s)\left(F_{n}\left(x_{n}\right)(s)-F_{n}(x)(s)\right)\right\|_{H} d s\right)^{2}
\end{gathered}
$$




$$
\left.\left.+\left(\int_{0}^{T}\left\|\int_{0}^{T} S_{n}(T-\tau)\left(G_{n}\left(x_{n}\right)(\tau)-G_{n}(x)(\tau)\right) d W(\tau)\right\|_{H} d s\right)^{2}\right]+\left\|y_{n}(t)-x(t)\right\|_{H}^{2}\right\} .
$$

Now, taking the expectation, followed by taking square roots, yields after some computation

$$
\begin{gathered}
\left\|x_{n}(t)-x(t)\right\|_{L^{2}(\Omega ; H)} \leq 2\left\{4 \left[E\left\|S_{n}(t)\left(h_{n}\left(x_{n}\right)-h_{n}(x)\right)\right\|_{L^{2}(\Omega ; H)}\right.\right. \\
+T^{1 / 2}\left(\int_{0}^{T} E\left\|S_{n}(T-s)\left(F_{n}\left(x_{n}\right)(s)-F_{n}(x)(s)\right)\right\|_{H}^{2} d s\right)^{1 / 2} \\
\left.+T^{1 / 2}\left(\int_{0}^{T} E\left\|\int_{0}^{T} S_{n}(T-\tau)\left(G_{n}\left(x_{n}\right)(\tau)-G_{n}(x)(\tau)\right) d W(\tau)\right\|_{H}^{2} d s\right)^{1 / 2}\right] \\
\left.+\left\|y_{n}(t)-x(t)\right\|_{L^{2}(\Omega ; H)}\right\} .
\end{gathered}
$$

For convenience, we relabel the first three terms on the right-side of (5.3) as $I_{1}, I_{2}$ and $I_{3}$, respectively, and estimate each separately below.

First, note that (H23) immediately yields

$$
I_{1} \leq \overline{M_{S}}\left\|h_{n}\left(x_{n}\right)-h_{n}(x)\right\|_{L_{0}^{2}} \leq \overline{M_{S}} M_{h}\left\|x_{n}-x\right\|_{\mathcal{C}} .
$$

Next, (H21) yields, with the help of Hölder's inequality,

$$
I_{2} \leq T^{1 / q} \overline{M_{S}}\left\|F_{n}\left(x_{n}\right)-F_{n}(x)\right\|_{L^{p}} \leq T^{1 / q} \overline{M_{S}} M_{F}\left\|x_{n}-x\right\|_{\mathcal{C}} .
$$

Finally, using (H22), we obtain

$$
\begin{gathered}
I_{3} \leq T^{1 / 2} \overline{M_{S}}\left(\int_{0}^{T} \int_{0}^{T}\left\|G_{n}\left(x_{n}\right)(\tau)-G_{n}(x)(\tau)\right\|_{L^{2}(\Omega ; B L(K ; H))}^{2} d \tau d s\right)^{1 / 2} \\
\leq T^{5 / 2} \overline{M_{S}}\left\|G_{n}\left(x_{n}\right)-G_{n}(x)\right\|_{\mathcal{C}_{B L}} \\
\leq T^{5 / 2} \overline{M_{S}} M_{G}\left\|x_{n}-x\right\|_{\mathcal{C}} .
\end{gathered}
$$

Using (5.4)-(5.6) in (5.3) yields, after taking supremum over $[0, T]$,

$$
1 / 2\left(1-8 \overline{M_{S}}\left[M_{h}+T^{1 / q} M_{F}+T^{5 / 2} M_{G}\right]\right)\left\|x_{n}-x\right\|_{\mathcal{C}} \leq\left\|y_{n}-x\right\|_{\mathcal{C}} .
$$

In view of $(\mathrm{H} 20)-(\mathrm{H} 23)$, and the fact that $1-8 \overline{M_{S}}\left[M_{h}+T^{1 / q} M_{F}+T^{5 / 2} M_{G}\right]>0$, we can apply Lemma 5.1 to conclude from (5.7) that $x_{n} \stackrel{\mathcal{C}}{\rightarrow} x$ as $n \rightarrow \infty$.

Now, let $P_{x}$ and $P_{x_{n}}$ denote the probability measures on $\mathcal{C}([0, T] ; H)$ induced by the mild solutions $x$ and $x_{n}$ of (1.1) and (5.1), respectively. Using Theorem 5.2, we can prove that $P_{x_{n}} \stackrel{w}{\rightarrow} P_{x}$ as $n \rightarrow \infty$, for a certain subclass of perturbations. Precisely, we have

Theorem 5.3:Let $p \geq 4$ and assume that $S_{n}(\cdot) A_{n}$ is a bounded operator, for each $n \in \mathbb{N}$. Then, $P_{x_{n}} \stackrel{w}{\rightarrow} P_{x}$ as $n \rightarrow \infty$, provided that

(H24) $1-{\overline{M_{S}}}^{2}\left[M_{h}^{2}+T^{2 / q}{\bar{M}_{F}}^{2}+C_{G}^{3} T^{3} M_{G}^{2}\right]>0$. 
Proof: We shall employ a standard argument involving Theorem 2.6 similar to the one used in [22].

We begin by showing $\left\{P_{x_{n}}\right\}_{n=1}^{\infty}$ is relatively compact in $\mathcal{C}([0, T] ; H)$ by appealing to the Arzelá-Ascoli theorem. To this end, we shall first show that there exists $\eta>0$ such that

$$
\sup _{n \in \mathbb{N}} \sup _{0 \leq t \leq T}\left\|x_{n}(t)\right\|_{L^{2}(\Omega ; H)}=\eta<\infty .
$$

Note that $x_{n}$ is given by

$$
\begin{gathered}
x_{n}(t)=S_{n}(t)\left(h_{n}\left(x_{n}\right)+x_{0}\right)+\int_{0}^{t} S_{n}(t-s) F_{n}\left(x_{n}\right)(s) d s \\
+\int_{0}^{t} \int_{0}^{s} S_{n}(s-\tau) G_{n}\left(x_{n}\right)(\tau) d W(\tau) d s, 0 \leq t \leq T .
\end{gathered}
$$

Since $h_{n}(0) \stackrel{L_{0}^{2}}{\rightarrow} h(0)$, there exists $\overline{M_{h}}>0$ (independent of $n$ ) such that $\left\|h_{n}(0)\right\|_{L_{0}^{2}} \leq \overline{M_{h}}$, for all $n$. Using this fact, together with (H20) and (H23)(i), we arrive at

$$
\left\|S_{n}(t)\left(h_{n}\left(x_{n}\right)+x_{0}\right)\right\|_{L^{2}(\Omega ; H)}^{2} \leq{\overline{M_{S}}}^{2} M_{h}^{2}\left\|x_{n}\right\|_{\mathcal{C}}^{2}+{\overline{M_{S}}}^{2}\left[{\overline{M_{h}}}^{2}+\left\|x_{0}\right\|_{L_{0}^{2}}^{2}\right]
$$

Likewise, (H21)(ii) and (H22)(ii) guarantee that there exist $\overline{M_{F}}, \overline{M_{G}}>0$ such that $\left\|F_{n}(0)\right\|_{L^{p}} \leq \overline{M_{F}}$ and $\left\|G_{n}(0)\right\|_{\mathcal{C}_{B L}} \leq \overline{M_{G}}$, for all $n$, so that a standard argument now yields

$$
E\left\|\int_{0}^{t} S_{n}(t-s) F_{n}\left(x_{n}\right)(s) d s\right\|_{H}^{2} \leq T^{2 / q}{\overline{M_{S}}}^{2}\left[M_{F}^{2}\left\|x_{n}\right\|_{\mathcal{C}}^{2}+{\overline{M_{F}}}^{2}\right]
$$

and

$$
E\left\|\int_{0}^{t} \int_{0}^{s} S_{n}(s-\tau) G_{n}\left(x_{n}\right)(\tau) d W(\tau) d s\right\|_{H}^{2} \leq T^{3}{\overline{M_{S}}}^{2} C_{G}^{2}\left[M_{G}^{2}\left\|x_{n}\right\|_{\mathcal{C}}^{2}+{\overline{M_{G}}}^{2}\right] .
$$

Combining the estimates (5.10)-(5.12) and rearranging terms, we can now conclude from (5.9) that (5.8) holds due to (H24) and the fact that all constants in (5.10)-(5.12) are independent of $n$.

Next, we establish the equicontinuity by showing $E\left\|x_{n}(t)-x_{n}(s)\right\|_{H}^{4} \rightarrow 0$ as $(t-s) \rightarrow$ 0 , for all $0 \leq s \leq t \leq T$, uniformly for all $n \in \mathbb{N}$. We estimate each term of the representation formula for $x_{n}(t)-x_{n}(s)$ (cf. (5.9)) separately. Employing Theorem 2.4(d) in [30] and taking into account (H20), (H23), and the uniform boundedness of $S_{n}(\cdot) A_{n}$, we conclude that

$$
\begin{gathered}
E\left\|\left[S_{n}(t)-S_{n}(s)\right]\left(h_{n}\left(x_{n}\right)+x_{0}\right)\right\|_{H}^{4} \leq T^{4 / 3} \int_{s}^{t} E\left\|S_{n}(\tau) A_{n}\left(h_{n}\left(x_{n}\right)+x_{0}\right)\right\|_{H}^{4} d \tau \\
\leq T^{4 / 3}\left\{M_{S A}\left[M_{h}^{2}\left\|x_{n}\right\|_{\mathcal{C}}^{2}+{\overline{M_{h}}}^{2}\right]+{\overline{M_{S}}}^{2}\left\|x_{0}\right\|_{L_{2}^{0}}^{2}\right\}(t-s)^{2}
\end{gathered}
$$

where $M_{S A}=\sup _{n \in \mathbb{N}}\left\|S_{n}(\cdot) A_{n}\right\|_{B L}$. Next, note that

$$
\int_{0}^{t} S_{n}(t-\tau) F_{n}\left(x_{n}\right)(\tau) d \tau-\int_{0}^{s} S_{n}(s-\tau) F_{n}\left(x_{n}\right)(\tau) d \tau
$$




$$
\begin{gathered}
=\int_{0}^{s}\left[S_{n}(t-\tau)-S_{n}(s-\tau)\right] F_{n}\left(x_{n}\right)(\tau) d \tau \\
\quad+\int_{s}^{t} S_{n}(t-\tau) F_{n}\left(x_{n}\right)(\tau) d \tau .
\end{gathered}
$$

Estimating each of the two integrals on the right-side of (5.14) separately yields, from the boundedness of $S_{n}(\cdot) A_{n},(\mathrm{H} 20)$, and (H21)(i), that

$$
\begin{aligned}
& E\left\|\int_{0}^{s}\left[S_{n}(t-\tau)-S_{n}(s-\tau)\right] F_{n}\left(x_{n}\right)(\tau) d \tau\right\|_{H}^{4} \\
\leq & T^{8 / 3} \int_{0}^{s} \int_{s-\tau}^{t-\tau} E\left\|S_{n}(w) A_{n} F_{n}\left(x_{n}\right)(w)\right\|_{H}^{4} d w d \tau \\
\leq & M_{S A}^{4} T^{11 / 3}\left[M_{F}^{4}\left\|x_{n}\right\|_{\mathcal{C}}^{4}+{\overline{M_{F}}}^{4}\right](t-s)^{(p-4) / p},
\end{aligned}
$$

and similarly,

$$
\begin{gathered}
E\left\|\int_{s}^{t} S_{n}(t-\tau) F_{n}\left(x_{n}\right)(\tau) d \tau\right\|_{H}^{4} \leq T^{2}{\overline{M_{S}}}^{4} \int_{s}^{t}\left\|F_{n}\left(x_{n}\right)(\tau)\right\|_{L^{2}(\Omega ; H)}^{4} d \tau \\
\leq T^{2}{\overline{M_{S}}}^{4}\left[M_{F}^{4}\left\|x_{n}\right\|_{\mathcal{C}}^{4}+{\overline{M_{F}}}^{4}\right](t-s)^{(p-4) / p} .
\end{gathered}
$$

Regarding the difference of the stochastic integrals, note that Fubini's theorem, together with basic integral properties, enables us to write

$$
\begin{gathered}
\int_{0}^{t} \int_{0}^{\tau} S_{n}(\tau-\theta) G_{n}\left(x_{n}\right)(\theta) d W(\tau) d \theta-\int_{0}^{s} \int_{0}^{\tau} S_{n}(\tau-\theta) G_{n}\left(x_{n}\right)(\theta) d W(\tau) d \theta \\
=\int_{0}^{s} \int_{s}^{\tau}\left[S_{n}(t-\theta)-S_{n}(s-\theta)\right] G_{n}\left(x_{n}\right)(\tau) d \theta d W(\tau) \\
+\left[\int_{0}^{s} \int_{s}^{t}+\int_{s}^{t} \int_{\tau}^{s}+\int_{s}^{t} \int_{s}^{t}\right] S_{n}(t-\theta) G_{n}\left(x_{n}\right)(\tau) d \theta d W(\tau) .
\end{gathered}
$$

Arguing as above, we see that

$$
\begin{gathered}
E\left\|\int_{0}^{s} \int_{s}^{\tau}\left[S_{n}(t-\theta)-S_{n}(s-\theta)\right] G_{n}\left(x_{n}\right)(\tau) d \theta d W(\tau)\right\|_{H}^{4} \\
\leq \int_{0}^{s} T^{4 / 3} E\left\|\int_{s}^{\tau} \int_{0}^{t-s} S_{n}(\mu+s-\theta) A_{n} G_{n}\left(x_{n}\right)(\tau) d W(\tau) d \theta\right\|_{H}^{4} d \mu \\
\leq T^{4 / 3} M_{S A} \int_{0}^{s} \int_{\tau}^{s} \int_{0}^{t-s}\left\|G_{n}\left(x_{n}\right)(\tau)\right\|_{B L}^{4} d \tau d \theta d \mu \\
\leq T^{4 / 3} M_{S A}\left[M_{G}^{4}\left\|x_{n}\right\|_{\mathcal{C}}^{4}+\bar{M}_{G}^{4}\right](t-s)^{4},
\end{gathered}
$$

and that

$$
E\left\|\left[\int_{0}^{s} \int_{s}^{t}+\int_{s}^{t} \int_{\tau}^{s}+\int_{s}^{t} \int_{s}^{t}\right] S_{n}(t-\theta) G_{n}\left(x_{n}\right)(\tau) d \theta d W(\tau)\right\|_{H}^{4}
$$




$$
\leq 3 T^{2}{\overline{M_{S}}}^{4}\left[M_{G}^{4}\left\|x_{n}\right\|_{\mathcal{C}}^{4}+{\overline{M_{G}}}^{4}\right](t-s)^{4} .
$$

Invoking (5.8) in (5.13), (5.15), (5.16), (5.18), and (5.19) enables us to conclude that, in fact, $E\left\|x_{n}(t)-x_{n}(s)\right\|_{H}^{4} \rightarrow 0$ as $(t-s) \rightarrow 0$, uniformly for $0 \leq s \leq t \leq T$ and $n \in \mathbb{N}$, as desired. Thus, the family $\left\{P_{x_{n}}\right\}_{n=1}^{\infty}$ is relatively compact in $\mathcal{C}([0, T] ; H)$ and hence, tight (by Prokorhov's theorem [11]).

To finish the proof, we remark that Theorem 5.2 implies that the finite-dimensional joint distributions of $P_{x_{n}}$ converge weakly to those of $P$ (cf. Proposition 2.5). Hence, Theorem 2.6 ensures that $P_{x_{n}} \stackrel{w}{\rightarrow} P_{x}$ as $n \rightarrow \infty$.

Remark: For the classical version of (5.1) (i.e., when $h_{n}=0$, for all $n$ ), a Gronwalltype argument can be used to establish the uniform boundedness (in $\mathcal{C}([0, T] ; H)$ ) of $\left\{x_{n}\right\}_{n=1}^{\infty}$ and, in such case, condition (H24) can be dropped.

\section{Example}

Let $\mathcal{D}$ be a bounded domain in $\mathbb{R}^{N}$ with smooth boundary $\partial \mathcal{D}$ and consider the initialboundary value problem

$$
\begin{gathered}
x_{t}(t, z)=\Delta_{z} x(t, z)+\int_{0}^{T} a(t, s) f_{1}\left(s, x(s, z), \int_{0}^{s} k(s, \tau, x(\tau, z)) d \tau\right) d s \\
+\int_{0}^{T} b(t, s) f_{2}(s, x(s, z)) d W(s), \text { a.e. on }(0, T) \times \mathcal{D}, \\
x(0, z)=\sum_{i=1}^{n} g_{i}(z) x\left(t_{i}, z\right)+\int_{0}^{T} c(s) f_{3}(s, x(s, z)) d s, \text { a.e. on } \mathcal{D}, \\
x(t, z)=0, \quad \text { a.e. on }(0, T) \times \partial \mathcal{D},
\end{gathered}
$$

where $0 \leq t_{1}<t_{2}<\ldots<t_{n} \leq T$ are given and $W$ is an $L^{2}(\mathcal{D})$-valued Wiener process (see [14] for examples). We consider (6.1) under the following conditions on the data:

(H25) $f_{1}:[0, T] \times \mathbb{R} \times \mathbb{R} \rightarrow \mathbb{R}$ satisfies the Caratheódory conditions (i.e., measurable in $(t, x)$ and continuous in the third variable), as well as

(i) $f_{i}(\cdot, 0,0) \in L^{2}(0, T)$,

(ii) $\left|f_{1}\left(t, x_{1}, y_{1}\right)-f_{1}\left(t, x_{2}, y_{2}\right)\right| \leq M_{f_{1}}\left[\left|x_{1}+x_{2}\right|+\left|y_{1}-y_{2}\right|\right]$, for all $x_{1}, x_{2}, y_{1}$, $y_{2} \in \mathbb{R}$ and almost all $t \in(0, T)$, for some $M_{f_{1}}>0$,

(H26) $f_{2}:[0, T] \times \mathbb{R} \rightarrow B L\left(L^{2}(\mathcal{D})\right)$ satisfies the Caratheódory conditions (cf. H(12) (i),

(ii)), as well as

(i) $f_{2}(\cdot, 0) \in L^{2}(0, T)$,

(ii) $\left|f_{2}(t, x)-f_{2}(t, y)\right|_{B L(H)} \leq M_{f_{2}}|x-y|$, for all $x, y \in \mathbb{R}$ and almost all $t \in$ $(0, T)$, for some $M_{f_{2}}>0$.

(H27) $f_{3}:[0, T] \times \mathbb{R} \rightarrow \mathbb{R}$ satisfies the Caratheódory conditions (cf. H(12) (i), (ii)), as well as

(i) $f_{3}(\cdot, 0) \in L^{2}(0, T)$, 
(ii) $\left|f_{3}(t, x)-f_{3}(t, y)\right| \leq M_{f_{3}}|x-y|$, for all $x, y \in \mathbb{R}$ and almost all $t \in(0, T)$, for some $M_{f_{3}}>0$,

(H28) $a \in L^{2}\left((0, T)^{2}\right)$,

(H29) $b \in L^{\infty}\left((0, T)^{2}\right)$,

(H30) $c \in L^{2}(0, T)$,

(H31) $k: U \times \mathbb{R} \rightarrow \mathbb{R}$, where $U=\{(s, t): 0<s<t<T\}$ satisfies

$$
\left|k\left(t, s, x_{1}\right)-k\left(t, s, x_{2}\right)\right| \leq M_{k}\left|x_{1}-x_{2}\right|,
$$

for all $x_{1}, x_{2} \in \mathbb{R}$, and almost all $(s, t) \in U$,

(H32) $g_{i} \in L^{2}(\mathcal{D}), i=1, \ldots, n$.

Let $H=K=L^{2}(\mathcal{D})$ and set

$$
A=\Delta_{z}, \quad D(A)=H^{2}(\mathcal{D}) \cup H_{0}^{1}(\mathcal{D}) .
$$

It is well-known that $A$ generates a $C_{0}$-semigroup on (see [30], Chapter 7). Next, define $F: \mathcal{C}([0, T] ; H) \rightarrow L^{2}\left(0, T ; L^{2}(\Omega ; H)\right), G: \mathcal{C}([0, T] ; H) \rightarrow \mathcal{C}_{B L}$, and $h: \mathcal{C}([0, T] ; H) \rightarrow$ $L_{0}^{2}(\Omega ; H)$, respectively, by

$$
\begin{gathered}
F(x)(t, \cdot)=\int_{0}^{T} a(t, s) f_{1}\left(s, x(s, \cdot), \int_{0}^{s} k(s, \tau, x(\tau, \cdot)) d \tau\right) d s \\
G(x)(s, \cdot)=a(t, s) f_{2}(s, x(s, \cdot)), \\
h(x)(\cdot)=x(0, z)=\sum_{i=1}^{n} g_{i}(\cdot) x\left(t_{i}, \cdot\right)+\int_{0}^{T} c(s) f_{3}(s, x(s, \cdot)) d s .
\end{gathered}
$$

One can use (H25)-(H32) to verify that $F, G$, and $h$ satisfy (H2)-(H4), respectively, with

$$
\begin{gathered}
M_{F}=2 M_{f_{1}} T|a|_{L^{2}\left((0, T)^{2}\right)}\left(1+M_{k_{1}} T^{3}\right)^{1 / 2}, \\
M_{G}=|b|_{L^{\infty}\left((0, T)^{2}\right)} M_{f_{2}}, \\
M_{h}=2\left(\sum_{i=1}^{n}\left\|g_{i}\right\|_{L^{2}(\mathcal{D})}+M_{f_{3}} \sqrt{m(\mathcal{D})}|G|_{L^{2}(0, T)}\right)
\end{gathered}
$$

where $m(\mathcal{D})$ is the Lebesgue product measure on $\mathcal{D}$. Thus, (6.1) can be rewritten in the form (1.1) in $H$, with $A, F, G$, and $h$ given by (6.2)-(6.5) so that, once (3.2) holds, an application of Theorem 3.2 immediately yields

Theorem 6.1: Assume (H25)-(H32) are satisfied. If, in addition, (3.2) holds (with $M_{F}, M_{G}$, and $M_{h}$ and given by (6.6)-(6.8)), then (6.1) has a unique mild solution $x \in C\left([0, T] ; L^{2}\left(\Omega ; L^{2}(\mathcal{D})\right)\right.$. 


\section{References}

[1] Ahmed, N.U., Differential inclusions on Banach spaces with nonlocal state constraints, Nonlinear Funct. Anal. \&S Appl. 6:3 (2001), 395-409.

[2] Aizicovici, S. and Gao, Y., Functional differential equations with nonlocal initial conditions, J. Appl. Math. Stochastic Anal. 10 (1997), 145-156.

[3] Aizicovici, S. and Hannsgen, K.B., Local existence for abstract semilinear Volterra integrodifferential equations, J. Integral Eqns. Appl. 5:3 (1993), 299-313.

[4] Aizicovici, S. and McKibben, M., Existence results for a class of abstract nonlocal Cauchy problems, Nonlinear Anal. 39:5 (2000), 649-668.

[5] Aizicovici, S. and McKibben, M., Semilinear Volterra integrodifferential equations with nonlocal initial conditions, Abstract \& Appl. Anal. 4:2 (1999), 127-139.

[6] Altman, M., Contractors and Contractor Directions, Theory and Applications, MarcelDekker, New York 1978.

[7] Balasubramaniam, P. and Ntouyas, S.K., Global existence for semilinear stochastic delay evolution equations with nonlocal conditions, Soochow Jour. of Math. 27:3 (July 2001), 331-342.

[8] Barbu, V., Nonlinear Semigroups and Differential Equations in Banach Spaces, Noordhoff, Leyden 1976.

[9] Bergström, H., Weak Convergence of Measures, Academic Press, New York 1982.

[10] Bharucha-Reid, A.T., Random Integral Equations, Academic Press, New York 1972.

[11] Billingsley, P., Weak Convergence of Measures: Applications in Probability, SIAM, Bristol 1971.

[12] Breiman, L., Probability, SIAM, Philadelphia 1992.

[13] Byszewski, L., Theorems about the existence and uniqueness of solutions of a semilinear evolution nonlocal Cauchy problem, J. Math. Anal. \&6 Appl. 162 (1991), 494-505.

[14] DaPrato, G. and Zabczyk, J., Stochastic Equations in Infinite Dimensions, Cambridge University Press, Cambridge 1992.

[15] Dunford, N. and Schwarz, J.T., Linear Operators, Part I, Wiley Interscience, New York 1958.

[16] Govindan, T.E., Autonomous semilinear stochastic Volterra integrodifferential equations in Hilbert spaces, Dyn. Sys. Appl. 3 (1994), 51-74.

[17] Govindan, T.E. and Joshi, M.C., Stability and optimal control of stochastic functional differential equations with memory, Numer. Func. Anal. Optim. 13 (1992), 249-265.

[18] Govindan, T.E., Stability of stochastic differential equations in a Banach space, In: Mathematical Theory of Control Lecture Notes in Pure and Applied Mathematics 142, MarcelDekker, New York 1992.

[19] Grecksch, W. and Tudor, C., Stochastic Evolution Equations: A Hilbert Space Approach, Akademic Verlag, Berlin 1995.

[20] Ichikawa, A., Stability of semilinear evolution equations, J. Math. Anal. Appl. 90 (1982), $12-44$.

[21] Jackson, D., Existence and uniqueness of solutions to semilinear nonlocal parabolic equations, J. Math. Anal. Appl. 172 (1993), 256-265.

[22] Kannan, D. and Bharucha-Reid, A.T., On a stochastic integrodifferential evolution equation of Volterra type, J. Integral Equations 10 (1985), 351-379. 
[23] Kunita, H., Stochastic Flows and Stochastic Differential Equations, Cambridge University Press, United Kingdom 1990.

[24] Leray, J. and Schauder, J., Topologie et equation fonctionelles, Ann. Sci. Ecole Norm. Sys. 51 (1934), 45-78.

[25] Lin, Y.P. and Liu, J.H., Semilinear integrodifferential equations with nonlocal Cauchy problems, Nonlinear Anal. 26:5, (1996), 1023-1033.

[26] Londen, S.O. and Nohel, J.A., Nonlinear Volterra integrodifferential equation occurring in heat flow, J. Integral Equations 6 (1984), 11-50.

[27] Ntouyas, S.K. and Tsamatos, P.Ch., Global existence for semilinear evolution equations with nonlocal conditions, J. Math. Anal. Appl. 210 (1997), 679-687.

[28] Ntouyas, S.K. and Tsamatos, P.Ch., Global existence for semilinear evolution integrodifferential equations with delay and nonlocal conditions, Appl. Anal. 64 (1997), 99-105.

[29] McKibben, M., Existence Results for Nonlinear Functional Differential Equations, Ph.D. dissertation, Ohio University, Athens, OH 1999.

[30] Pazy, A., Semigroups of Linear Operators and Applications to Partial Differential Equations, Springer-Verlag, New York 1983.

[31] Schaefer, H., Über die methode der a priori shranken, Math. Annal. 129 (1955), 415-416.

[32] Sobczyk, K., Stochastic Differential Equations with Applications to Physics and Engineering, Klüwer Academic Publishers, London 1991.

[33] Ward, J.R., Boundary value problems for differential equations in Banach space, J. Math. Anal. Appl. 70 (1979), 589-598.

[34] Zeidler, E., Nonlinear Functional Analysis and its Applications II/B: Nonlinear Monotone Operators, Springer, New York 1990. 


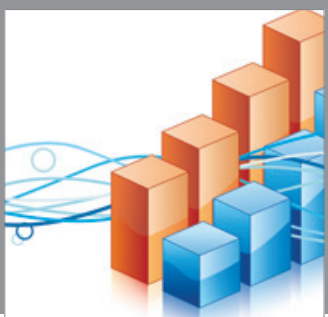

Advances in

Operations Research

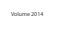

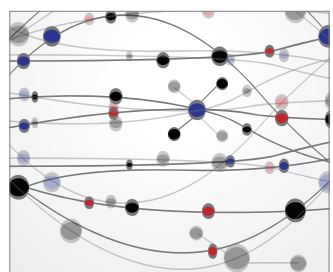

\section{The Scientific} World Journal
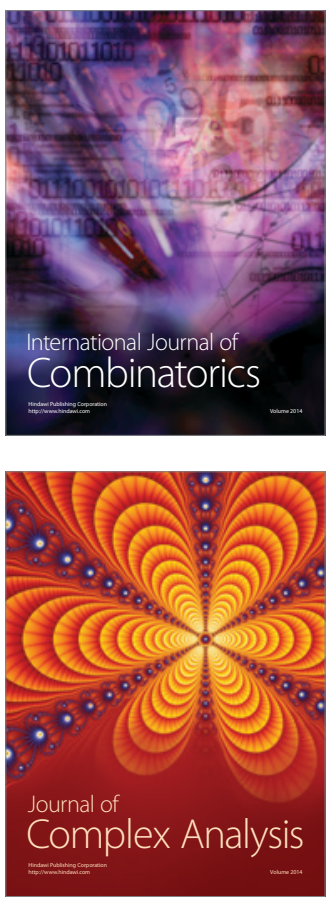

International Journal of

Mathematics and

Mathematical

Sciences
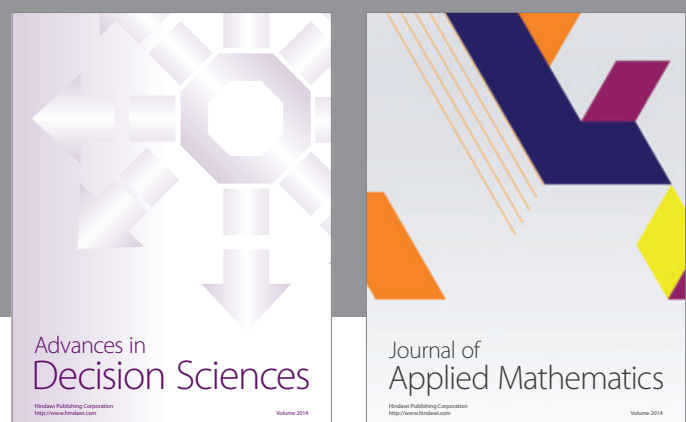

Journal of

Applied Mathematics
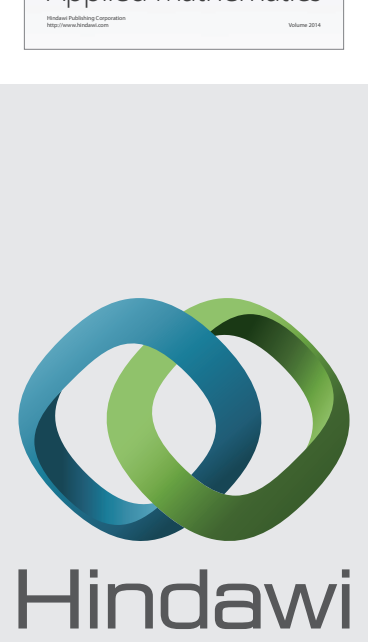

Submit your manuscripts at http://www.hindawi.com
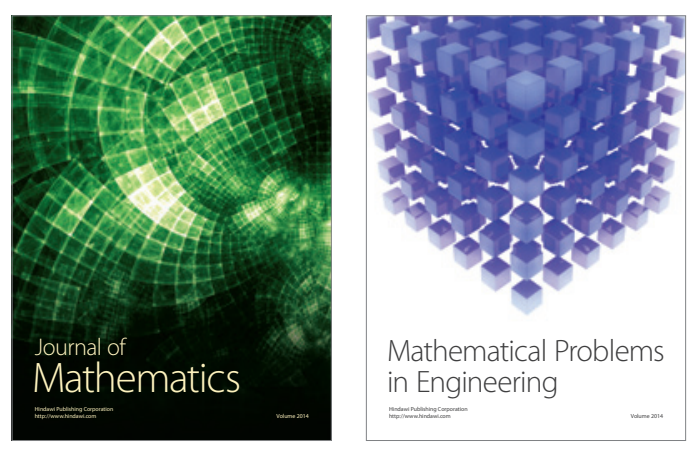

Mathematical Problems in Engineering
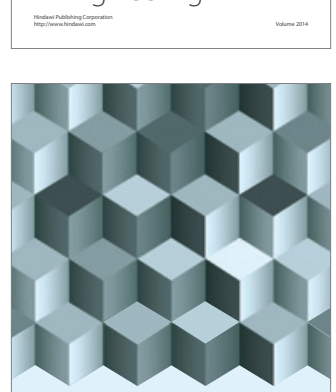

Journal of

Function Spaces
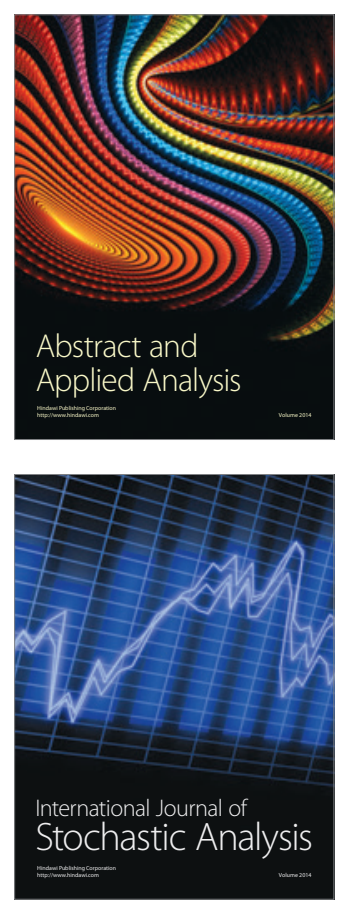

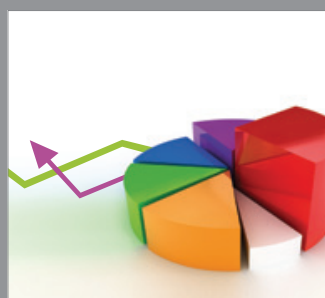

ournal of

Probability and Statistics

Promensencen
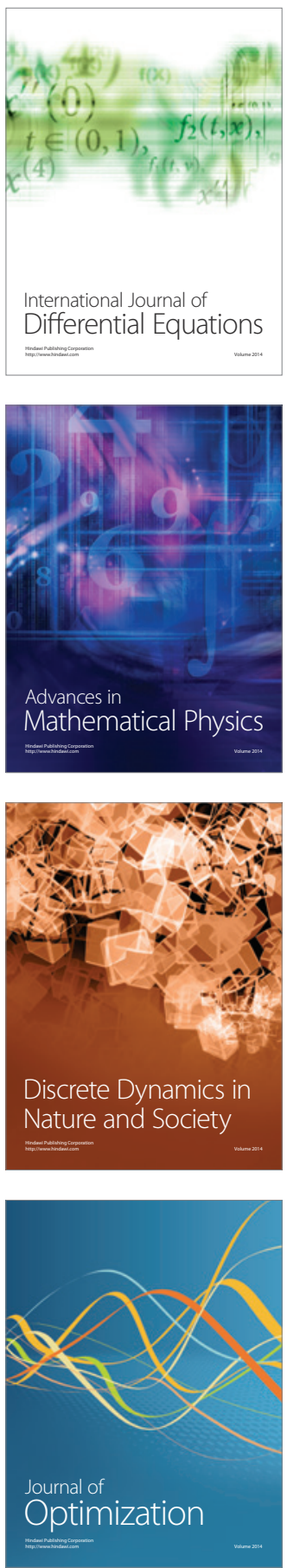